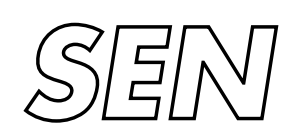

Software Engineering

$\int$ SEN $\begin{aligned} & \text { Software ENgineering } \\ & \text { Crosscutting concerns in J2EE applications }\end{aligned}$

A. Mesbah, A. van Deursen

Report SeN-R0513 September 2005 
$\mathrm{CWI}$ is the National Research Institute for Mathematics and Computer Science. It is sponsored by the Netherlands Organization for Scientific Research (NWO).

$\mathrm{CWI}$ is a founding member of ERCIM, the European Research Consortium for Informatics and Mathematics.

CWI's research has a theme-oriented structure and is grouped into four clusters. Listed below are the names of the clusters and in parentheses their acronyms.

Probability, Networks and Algorithms (PNA)

\section{Software Engineering (SEN)}

Modelling, Analysis and Simulation (MAS)

Information Systems (INS)

Copyright (C) 2005, Stichting Centrum voor Wiskunde en Informatica

P.O. Box 94079, 1090 GB Amsterdam (NL)

Kruislaan 413, 1098 SJ Amsterdam (NL)

Telephone +31205929333

Telefax +31205924199

ISSN 1386-369X 


\title{
Crosscutting concerns in J2EE applications
}

\begin{abstract}
We explore the evolution benefits of adopting aspects in a J2EE setting by studying crosscutting concerns in a typical J2EE application. To identify these concerns, we take a top-down as well as a bottom-up approach. In the top-down view we focus on typical concerns that are known to be crosscutting (e.g., persistence), the way they are currently implemented and the possible gains and benefits if solved by aspects. In the bottom-up approach we take a look at the application's source code, and apply aspect mining techniques in order to find cross cutting concerns. We discuss how such concerns can be represented in an aspect-oriented language (viz. AspectJ), and reflect on the results in terms of maintainability and evolvability of the affected system.
\end{abstract}

2000 Mathematics Subject Classification: 2000

1998 ACM Computing Classification System: D.2.10; D.2.7; D.2.3

Keywords and Phrases: cross cutting concerns; web application evolution; aspect-oriented

Note: This work was carried out under project SEN1 - Single Page Computer Interaction 



\title{
Crosscutting Concerns in J2EE Applications
}

\author{
Ali Mesbah, Arie van Deursen* \\ Centrum voor Wiskunde en Informatica \\ P.O. Box 94079, 1090 GB Amsterdam \\ The Netherlands \\ \{Ali.Mesbah, Arie.van.Deursen\}@Cwi.nl
}

\begin{abstract}
We explore the evolution benefits of adopting aspects in a J2EE setting by studying crosscutting concerns in a typical J2EE application. To identify these concerns, we take $a$ top-down as well as a bottom-up approach. In the topdown view we focus on typical concerns that are known to be crosscutting (e.g., persistence), the way they are currently implemented and the possible gains and benefits if solved by aspects. In the bottom-up approach we take a look at the application's source code, and apply aspect mining techniques in order to find crosscutting concerns. We discuss how such concerns can be represented in an aspect-oriented language (viz. AspectJ), and reflect on the results in terms of maintainability and evolvability of the affected system.
\end{abstract}

\section{Introduction}

From its inception, Java 2, Enterprise Edition (J2EE) ${ }^{1}$ has established a new model for developing distributed applications. It is based on well-defined components to provide server-side and client-side support for developing multi-tier applications. The J2EE architecture defines a client tier, a middle tier and a back-end tier, as depicted in Figure 1. The client tier provides support for a variety of client types (e.g. HTML pages generated by JavaServer Pages (JSP), Java applets, Java Web Start-enabled clients). The middle tier supports client services through Web containers (e.g. Servlets, JSP) and supports business logic component services through Enterprise JavaBeans (EJB) containers. The middle tier is often subdivided into the Web tier and EJB tier. On the back-end tier, the enterprise information systems are accessible by the way of standard APIs (e.g. JDBC).

Aspect-oriented software development (AOSD) [12] aims at improving the modularity of software systems, by capturing inherently scattered functionality, often called crosscutting concerns, in a well-modularized way, making the evo-

\footnotetext{
*Also affi liated at Delft University of Technology, Faculty of Electrical Engineering, Mathematics and Computer Science, Mekelweg 4, 2628 CD Delft, The Netherlands.

${ }^{1}$ http://java.sun.com/j2ee/
}

lution of such systems easier and manageable. In order to achieve this, aspect-oriented programming languages add an extra abstraction mechanism, called an aspect, on top of existing modularization mechanisms such as functions, classes and methods. Aspects allow developers to tackle the problems of scattering and tangling by reducing the spread of code belonging to a certain concern over different components.

J2EE already provides a transparent component-based framework in which many crosscutting concerns such as security and authentication are dealt with in the container without requiring the developer to know the corresponding implementation. However, introducing AOSD into J2EE application development could provide us with more flexible, modularized applications thus empowering the long-term evolution of applications developed in the framework.

The goal of this paper is to explore the evolution benefits of adopting aspects in a J2EE setting. To that end, we study crosscutting concerns in a typical J2EE application, Sun's Pet Store demonstration. To identify these concerns, we take a top-down as well as a bottom-up approach. In the topdown view we focus on typical concerns that are known to be crosscutting (e.g., persistence), the way they are currently implemented and the possible gains and benefits if solved by aspects. In the bottom-up approach we will take a look at the application's source code, and apply aspect mining techniques in order to find crosscutting concerns. We discuss how such concerns can be represented in an aspect-oriented language (viz. AspectJ), and reflect on the results in terms of maintainability and evolvability of the affected system.

This paper is laid out as follows. Section 2 discusses related work. In section 3 a short overview of the Pet Store web application is outlined which is the case study used in this paper. Section 4 discusses the top-down concerns and the ways they could be implemented by aspects. The bottom-up approach is presented in section 5 . In section 6 we discuss the achieved results. Finally, Section 7 presents our conclusions and future work.

We assume the reader has basic knowledge of both J2EE and AspectJ. The reader is referred to $[21]$ and $[15,8]$ re- 


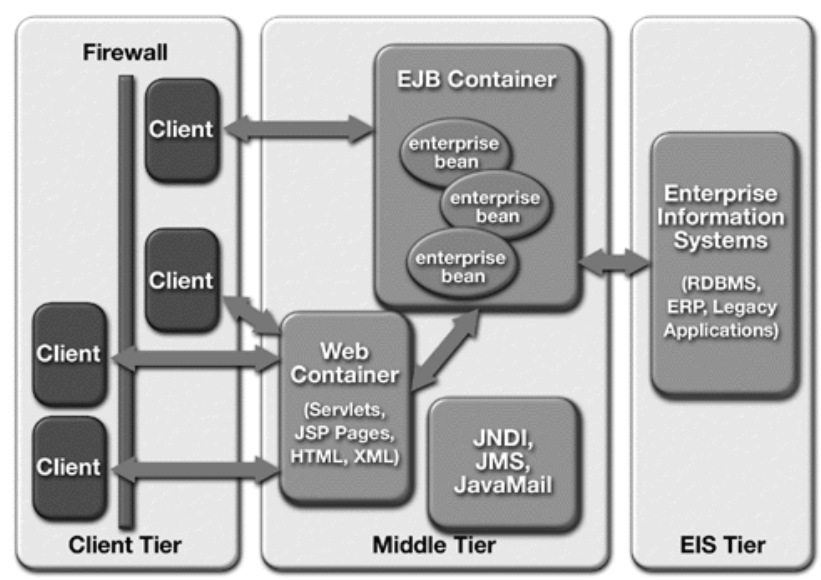

Figure 1. J2EE Environment [22]

spectively for more information on these topics.

\section{Related Work}

There are a number of publications reporting the possible applications of aspects to J2EE applications.

Soares et al. [24] discuss their experiences in applying the paradigm to a web-based information system's distribution, persistence and transaction concerns. Kerston et al. [11] share their experiences with a web-based educational system, separating network context concerns in a distributed environment. In [19], a component-based web-crawling system was developed with and without aspects to be able to evaluate the differences of both approaches. The results favor the aspect-oriented paradigm. Zhang and Jacobsen [26] apply aspect-oriented programming to middleware (Object Request Broker) demonstrating a reduction of the complexity of the architecture.

Kim and Clark [13] present a case study in which they show that using the EJB framework modularizes and minimizes crosscutting concerns such as security, persistence and transaction management. On the other hand, Choi [2] discusses the deficiencies of the EJB's architecture and tries to present an aspect-based server implementation.

A comparison of J2EE container managed and aspectoriented security is presented in [23]. Fabry [5] discusses the shortcomings of declarative transaction management in EJB with respect to a clean separation of concerns and proposes an aspect solution which can detect the need for transactional methods and integrate all transactional handling in one unit.

Han and Hofmeister [9] concentrate on navigation concerns of J2EE web applications and present an aspectoriented approach to separate these concerns.
An analysis of the crosscutting nature of the J2EE patterns is illustrated by [18].

Finally, Cohen and Gil [3] propose a promising new language, AspectJ2EE, geared towards the implementation of J2EE application servers and applications. They use a deploy-time mechanism to bind services to user applications in the application server. This would enable the EJB developers to extend, enhance and replace the standard services provided by the container.

None of these works however, cover how aspects influence evolution in J2EE environments, a gap we try to bridge in the present paper. Our work differs from these approaches in that

1. we adopt two different aspect mining techniques to identify candidate aspects;

2. we provide full details of the resulting aspect-oriented code, in order to provide a clear picture of the advantages and disadvantages; and

3. we explicitly evaluate the evolution benefits in terms of change scenarios.

\section{Pet Store}

Pet Store ${ }^{2}$ is an example of a J2EE web application developed by Sun in order to illustrate all sorts of J2EE features. The main function of the application is to allow customers to search and purchase pets through a web browser. The application provides the customers with an interface (Web site) to search through catalogs of products and order items. The Admin client has an interface to view sales statistics and manually accept or reject orders.

There are a number of reasons why we have chosen Pet Store as our case study. First of all, Pet Store is a welldesigned web application using the latest J2EE specifications. There are numerous design patterns used in its architecture such as MVC, ServiceLocator, Data Access Object, etc. It is a well-known open-source application. Further, even though the functionality of the system seems simple, the actual implementation is quite complex. The complexity is mainly due to the fact that it is a demo presenting the best and newest practices of J2EE application development. The complexity is also a price to pay for maintainability, portability and scalability of the application.

Finally, keeping all this in mind, our reasoning is based on the assumption that if we are able to identify crosscutting concerns and show benefits of aspectizing those concerns in such a well-designed system, then it should also be possible to find crosscutting concerns in real life J2EE applications, many of which are in practice not half as well-designed.

\footnotetext{
${ }^{2}$ http://java.sun.com/blueprints/, Java Pet Store Demo 1.3.2
} 


\section{Top-down concerns}

In this section we will consider a number of concerns that are know to be crosscutting such as Persistence, Transaction management and Security, from a top-down perspective. We will look at how each is implemented in J2EE environments, and whether the implementation can be improved if aspects are applied.

\subsection{Persistence}

Persistence is defined as the storage and retrieval of application data from secondary storage media (e.g databases) which can be considered a crosscutting concern [20] by the assumption that the application should remain oblivious of the concern during the development process.

There is a range of persistence architecture options available to J2EE developers. At one end there is the low-level Java Database Connectivity (JDBC). At the other end there is the high-level abstraction and integration with the EJB Container Managed Persistence (CMP) implementation. The most commonly used solutions include:

- Plain Old Java Objects (POJO) Persistence

- EJB Bean Managed Persistence (BMP)

- EJB Container Managed Persistence (CMP)

POJO is merely a fancy name given to normal traditional JavaBeans. It offers an alternative for those who need persistence capabilities but do not want the associated costs of CMP and BMP beans. The persistence is managed programmatically by the developers or it is handled by third party solutions such as Hibernate.

BMP developers create entity beans that are managed by the J2EE container, but the persistence of these beans is managed programmatically by the bean developer. Although this puts the responsibility back on the developer, it does offer an EJB entity bean solution that enables portability across multiple application server vendors.

With CMP, the bean provider relies on the container to manage the access to the persistent state of the bean. Unlike the first two solutions, the bean provider does not have to write any programming code but uses the declarative descriptor to request persistence on the bean.

Pet Store uses CMP as well as BMP to manage persistence. All modules requiring persistence (e.g. Customer, Profile, Address) are implemented as CMP entity beans except for the Catalog module. Very little code is necessary to create these entity beans and no code is needed for persistence. As such, the crosscutting nature of persistence has been resolved by J2EE's CMP container mechanism.

Catalog module needs more attention as it is using BMP and therefore could be a possible aspect candidate. It is used to retrieve information about catalog entries (categories, products, and items), either individually or page-by-page. Because the catalog module is the most highly-used component in the application, it is more efficient to access it relationally instead of using entity beans which may incur a performance penalty while providing little or no additional value. This is the reason why Pet Store uses a bean-managed persistence approach for this module - a fairly common situation for handling persistence in many real life J2EE applications.

Catalog is implemented as a stateless session bean, using a Data Access Object (DAO) interface to encapsulate access to persistent data. The persistence data are modeled as POJO's. The DAO interface is implemented depending on the specific type of database used. This approach encapsulates the persistence concern in one implementing class of the DAO interface, thus diminishing the scattering of the concern in the system.

There is however one aspect which is required by the persistence concern even in this well designed approach. It is required from each individual item to have a unique identifier. This unique identifier is used to retrieve the item from the underlying storage media. This could be seen as a crosscutting concern as the persistence aspect is not transparent anymore because of this requirement.

To be able to achieve total obliviousness, we have aspectized the identifier of the data classes using an Ident ifiable interface and making all the data classes implement this interface using aspects as explained in 5.2.

\subsection{Transaction management}

Transaction management is one of the important concerns of distributed systems. It ensures that the system remains in a consistent state before and after its execution calls. Atomicity is a property of a transaction which makes sure that either all the operations succeed within an execution call as a single unit or if one of the operations fails, the system abandons the whole operation and rolls back to its initial state. Transaction management can be a crosscutting concern, spanning over multiple modules.

In J2EE applications, transaction management is handled in either a declarative or programmatic form.

EJB provides an elegant system for handling transaction management by enabling the developers to declare the transactional functionality in the EJB's deployment descriptor. It is then the container that has to take care of the actual implementation. This approach separates the transaction management from the core business logic of the application. As Fabry [5] discusses, one could manage to produce tangling and scattering code even when using the declarative possibilities of EJB if the functionality provided by the container is not satisfactory.

Transaction management is also used in other parts of the 


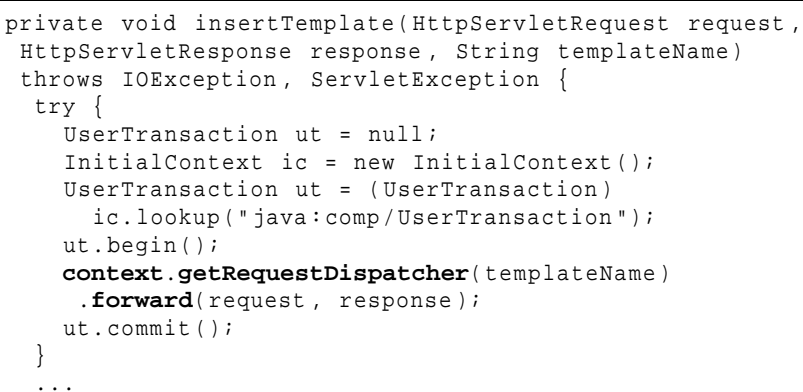

\section{Figure 2. Method using Transaction management}

J2EE platform (e.g. Servlets) where no EJB is present. J2EE does not provide any support for non-EJB parts regarding the declarative transactional management.

We analyzed the Pet Store application code looking for possible crosscutting transactional concerns. The application makes exclusive use of the declarative transaction control in the EJB tier without producing any tangling code. The EJB deployment descriptor defines any call to methods that need transactional support by setting the transaction attribute to Required.

In the Web tier a programmatic approach is used in two classes to manage transaction. The TemplateServlet begins a UserTransaction before it forwards a request to the template JSP and ends the transaction after the forward has accomplished. See Figure 2. The dopost method of the RcvrRequestProcessor Servlet uses the same transactional code to update supplier inventories. In order to be able to aspectize the transactional code, the doPost method had first to be refactored because it was used in a nested if statement. We moved the transactional handling into a new method called updateAndSend. This refactored method used the transactional code in a similar way as in Figure 2.

We decided to aspectize the transactional management in the following way [15]. First we defined an abstract aspect, which has an around advice while keeping the pointcut abstract . See Figure 3. Then we created a concrete transaction aspect which inherits from the abstract aspect, providing concrete definitions for the abstract point cut. See Figure 4.

With this aspectized approach, the transactional control code is modularized and is reusable. Further, the classes using the functionality are now oblivious of the transactional code.

\subsection{Security}

Security can be seen as the most important concern in J2EE applications. The system can usually be accessed by users from all around the globe and that makes authentication and authorization an essential part of the application. Security

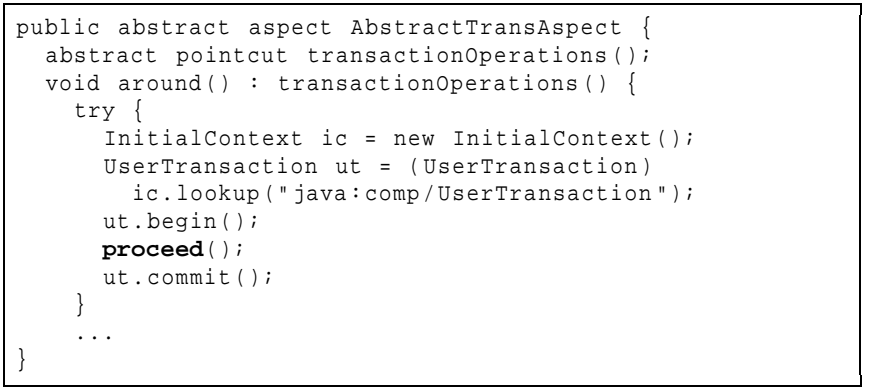

Figure 3. Abstract Transaction Aspect

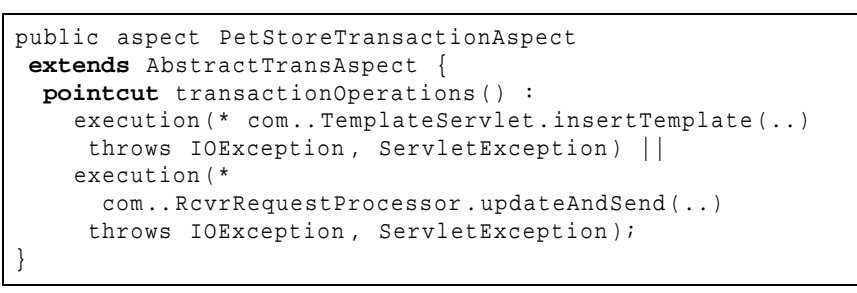

\section{Figure 4. Pet Store Transaction Aspect}

becomes a crosscutting concern because conventional programming techniques require us to modify modules individually to equip them with security code.

J2EE handles security in a much more modular way e.g. separating the security properties in the deployment descriptor.

Pet Store, however, does not use any of the security mechanisms specified by the J2EE platform. Instead, all requests to pages are first to go through a filter which checks whether the page requested is a protected one. If so, it checks to see if the user is signed-on. If that is not the case, the user is forwarded to the sign-on page. Upon receiving a sign-on request, the filter checks the username/password combination and creates and maintains a user entity bean.

In Pet Store, all the functionality needed for security is implemented in the signon module, leaving the system with no crosscutting security concern in other parts of the system. This is possible because security is only applied on a page request level.

For situations where crosscutting security concerns can be found in the system we refer to [23].

\subsection{Other top-down concerns}

There are also other relevant top-down concerns such as Tiercutting concerns (e.g. compression, encryption [3]), Logging and Exception Handling which are left out of this paper because of space limitations. The crosscutting nature of some of the J2EE patterns can also be examined from a top-down perspective as discussed in [18]. 


\section{Bottom-up concerns}

Aspect mining is the quest for identifying candidate aspects in existing object-oriented systems and isolating them into aspects, improving the comprehensibility of the system, and thereby improving it's maintainability and evolvability [4].

Aspect mining techniques can take a bottom-up approach in which they try to examine the current implementation of a software system, looking for patterns of tangling and scattering code which would make good aspect candidates. This bottom-up approach could also be applied to J2EE applications to find and extract crosscutting concerns.

In this section we will look at two aspect mining techniques and see how each technique could be used in J2EE environments discussing the possible benefits of applying each approach.

\subsection{Fan-In Analysis}

Fan-in analysis [17] takes a bottom-up approach, analyzing the code for certain crosscutting symptoms. This technique defines the number of distinct method calls to a given method as the fan-in number. Methods having a high fan-in value are likely to be crosscutting methods and should be further examined to see whether they could be considered as candidate aspects.

The Pet Store application code was analyzed by [17], identifying a number of candidate aspects for methods with a fan-in of 8 and above.

Next we will present an aspect-oriented implementation of the identified candidate aspects in order to discuss the effects the refactoring would have on the maintainability and evolvability of the application.

\subsubsection{Exception Wrapping}

Exception wrapping is a crosscutting concern that affects a number of classes in Pet Store. The ServiceLocator classes for instance catch the NamingException and rethrow ServiceLocatorException. This is a typical instance of the Business Delegate J2EE pattern [1]. Every method in these classes catches exceptions thrown by the underlying implementation and re-throws an applicationspecific exception requiring a try/catch block in each method. Normal refactoring of these identical try/catch blocks is not possible in object-oriented languages. This logic duplication is a result of a language limitation. Aspects can however be used to refactor this pattern of scattering code $[16,14]$.

To refactor the exception wrapping logic, we implement an aspect in which the checked-exceptions are declared soft. This way the exception will be wrapped in an uncheckedexception (SoftException) when thrown. An after throwing advice is then used to catch any SoftException thrown

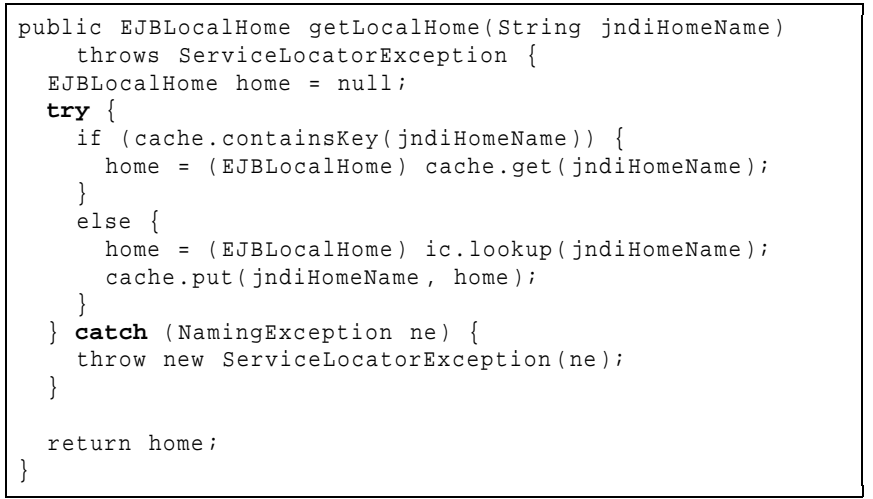

Figure 5. Exception wrapping in Pet Store

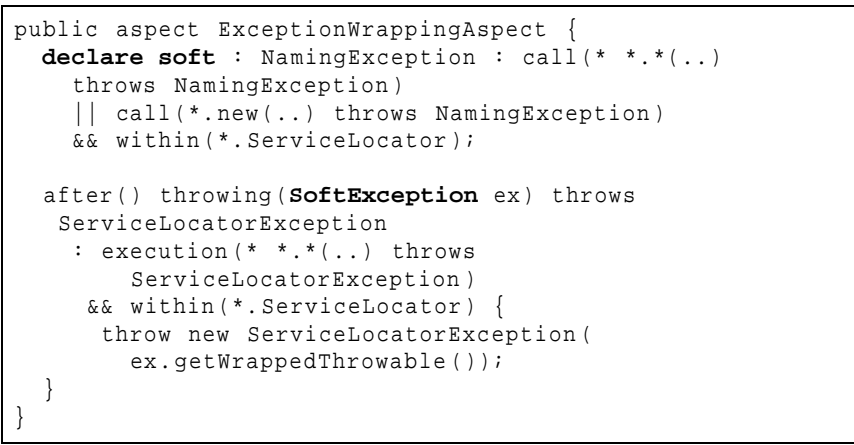

\section{Figure 6. Exception wrapping in AspectJ}

and throw a new ServiceLocatorException wrapping the original exception obtained.

Figure 5 shows one of the web ServiceLocator methods in its original form. Applying the aspect presented in Figure 6 would allow us to refactor the method (and all the other methods in the class). Figure 7 shows the refactored method.

As can be seen, the refactoring of the exception wrapping concern results in a simple aspect defining the wrapping strategy, and cleaner business logic that is not tangled with wrapping anymore. This not only leads to a reduction in code size of up to $20 \%$ in the refactored classes, it also improves evolvability of both the business logic and the wrapping strategy.

\subsubsection{ServiceLocator \& Singleton}

A ServiceLocator J2EE pattern [1] acts as a central point for obtaining and caching a service. This pattern implemented in classes ServiceLocator was also identified as a candidate aspect in Pet Store. There are two ServiceLocator classes in Pet Store one of which (JMS) is implemented as a Singleton and the other one (EJB) is (mistakenly) not. 


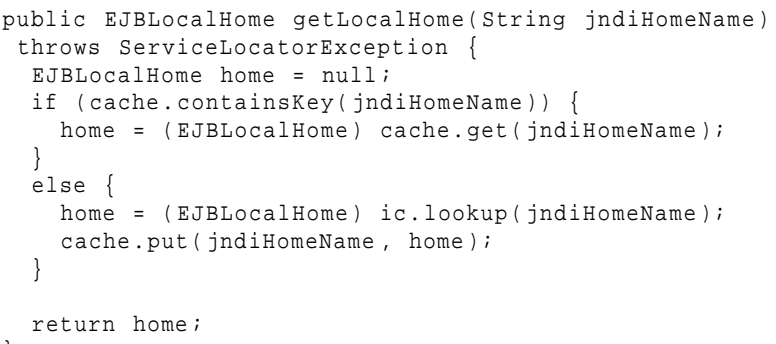

\section{Figure 7. Aspectized by ExceptionWrappingAspect}

The ServiceLocator can be approached in two ways. One could argue that because the getInstance method has a high fan-in, the methods calling the method should be refactored in a way that no explicit calling is needed. There are techniques which make this approach possible. For instance, Spring's ${ }^{3}$ inversion of control allows us to pass the ServiceLocator instance reference to our classes in a subtle manner. However, because of the non-systematic nature of the methods, it is not possible to create a unified aspect which would allow us to refactor all the calls to the get Instance method.

The second approach, presented by $[10,18]$, tries to refactor the Singleton nature of the ServiceLocator class itself. This means removing the private constructor and the get Instance of the class and instead providing a public constructor which is implemented as an around advice, initializing the instance on the first call and returning it on all constructor calls.

The Singleton class was refactored using the aspect shown in Figure 8. As can be seen, the aspect captures all calls to the constructor using the around advice. The advice then creates the static instance if not already created and initializes the object; otherwise the instance is returned without manipulation.

Now the question is what the consequences are of this refactoring step. Hiding the Singleton nature of the class could confuse J2EE developers. This could also lead to violations of the singleton nature, if, for example, a subclass provides cloning functionality. [18] and [10] mention a workaround for this possible problem.

On the other hand one could argue that hiding the Singleton nature of the class makes the application oblivious in the sense that the clients using the ServiceLocator class do not and should not care about the implementation details of the service.

\footnotetext{
${ }^{3}$ http://www.springframework.org/
}

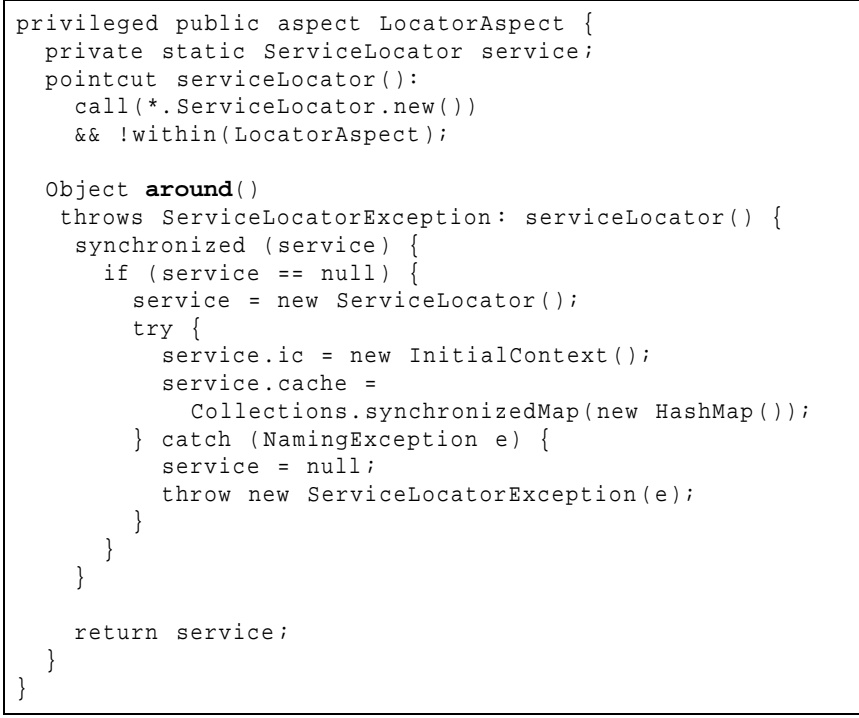

\section{Figure 8. Locator aspect}

\subsubsection{Precondition Checking}

Precondition checking often requires duplicated code if the conditions are common to many methods. In Pet Store many EJB classes implementing EntityBean use a Plain Old Java Object (POJO) of their own to hold the actual data. All these classes have a static fromDOM method which expects a DOM node as input parameter and returns an instance of the corresponding class made from the node. The node has to have a certain structure for the method to be able to parse it to the right class. The main precondition states that the name of the first element of the DOM has to coincide with the value of a static variable defined in the class. The Address class, for instance, has a static variable called XML_ADDRESS which has a string value of "Address". This means the first element of the DOM has to be called "Address", otherwise the check will through an XMLDocumentException.

The parameter checks occur at the beginning of the methods and are similar to:

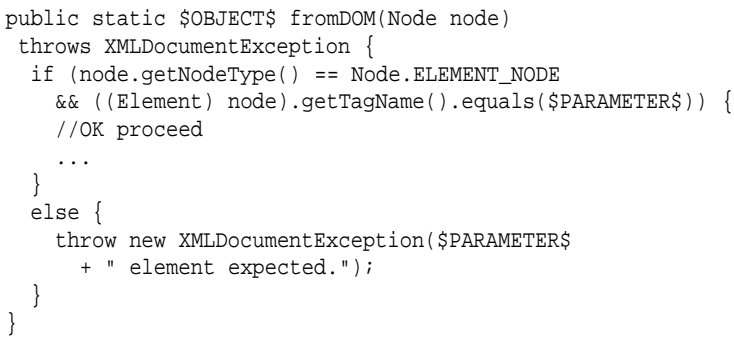

where \$OBJECT\$ is the corresponding class type (e.g. Address) and \$PARAMETER\$ is the name of the static variable holding the expected name of the first element. 


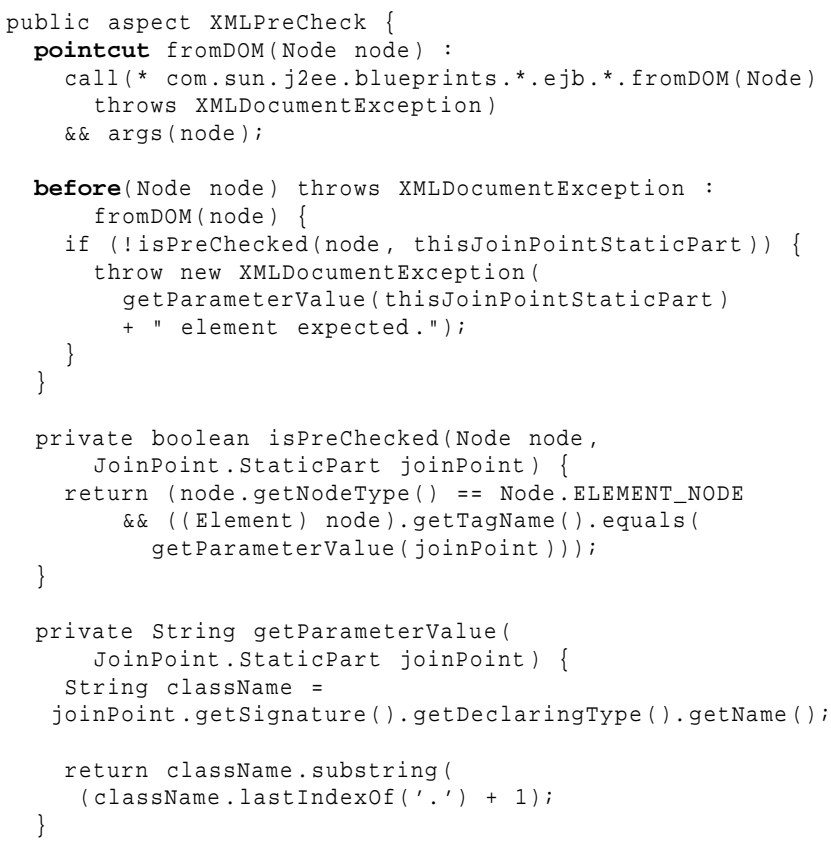

\section{Figure 9. Precondition Check Aspect}

Conventional implementations of this precondition require adding identical code conditional checks into many methods. With aspect-oriented techniques, we can refactor such contract checks into a separate aspect. Figure 9 shows the aspect which handles the precondition checks. We define the pointcut as calls to the fromDOM methods and use a before advice to check the precondition. The tricky part in this advice is to find the value of the expected variable on which the check has to be carried out. The thisJoinPointStaticPart gives us the corresponding class information and using reflection on the class, we can find the required parameter. By analyzing the code, a pattern can be seen in the value of the required variable; i.e., the value of the static variable is the same as the class-name. This means we can use the class-name to check our precondition and that is exactly what we have done. This, however, implicitly defines a contract by which all future classes of the same type have to abide.

Using this precondition aspect we are able to refactor out the precondition checks from nine classes in Pet Store.

\subsection{Interface Concerns}

Interfaces and their implementations can be crosscutting. When the implementation of an interface is distributed across many classes in a system, aspect-oriented programming enables us to extract and locate it in an aspect, increasing the modularity of the system. In order to investigate the behav-

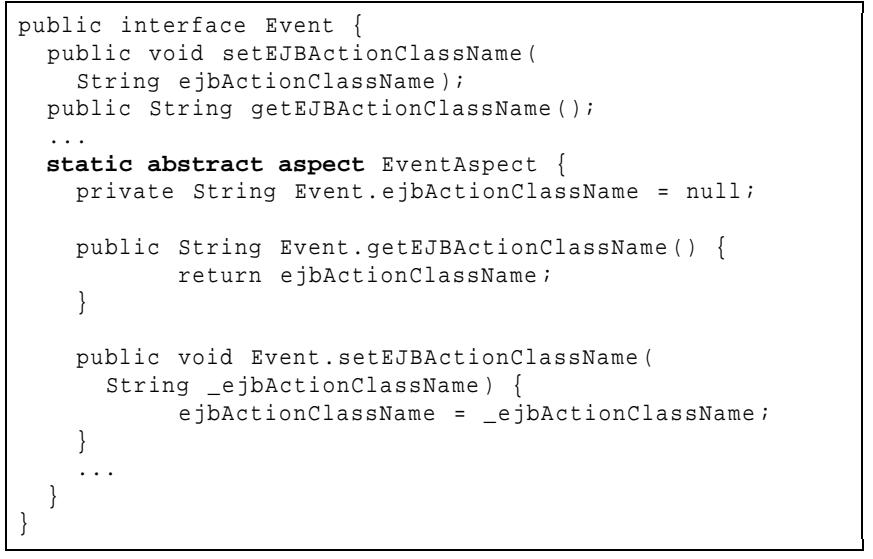

Figure 10. Aspectized Event interface

ior of this crosscutting concern in J2EE applications, we analyzed the Pet Store application, looking for possible aspect candidates. The results are discussed here.

\section{Extracting Interface Implementations}

The first concern that was identified as a possible aspect candidate was found because four interfaces (Event, EJBAction, HTMLAction and EventResponse) had a default implementation (EventSupport, EJBActionSupport, HTMLActionSupport and EventResponseSupport). Other classes then extended these support classes instead of implementing the interfaces. While Pet Store avoids duplicated code using this technique, this approach fails for the classes that are already extending another class or for classes that need to extend another class.

Using the refactoring technique suggested by [14], we are able to make use of the inter-type declaration mechanism to write aspects which introduce the default implementation into the identified interfaces.

Figure 10 shows the aspectized Event interface. The inner aspect functions as an implementation of the interface. This way the EventSupport class becomes obsolete. The main advantage of this refactoring is that all the classes which extended the EventSupport class can now implement the Event interface without the need to implement the declared methods in the interface (if they are satisfied with the default implementation of course) and more importantly, the classes can now extend another class if needed.

\section{Interface Migrating}

Interface migration tries to mine interface implementations which can be regarded as crosscutting concerns and refactor them to aspects. Tonnela et al. [25] represent a mining technique based on external package identification, string match- 


\begin{tabular}{|c|c|c|c|c|c|c|c|}
\hline Concern & Crosscutting & Aspectizable & Code Reduction & "Obliviousness & Reliability & Modularity & Evolvability \\
\hline Persistence & $\checkmark$ & $\checkmark$ & & $\checkmark$ & & & \\
\hline Transaction & $\checkmark$ & $\checkmark$ & $\checkmark$ & $\checkmark$ & $\checkmark$ & $\checkmark$ & $\checkmark$ \\
\hline Security & & & & & & & \\
\hline Exception Wrapping & $\checkmark$ & $\checkmark$ & $\checkmark$ & $\checkmark$ & $\checkmark$ & $\checkmark$ & $\checkmark$ \\
\hline ServiceLocator & $\checkmark$ & $\checkmark$ & & $\checkmark$ & & & $\checkmark$ \\
\hline Precondition Checking & $\checkmark$ & $\checkmark$ & $\checkmark$ & $\checkmark$ & $\checkmark$ & $\checkmark$ & $\checkmark$ \\
\hline Interface Extraction & $\checkmark$ & $\checkmark$ & & & & $\checkmark$ & $\checkmark$ \\
\hline Interface Migration & $\checkmark$ & $\checkmark$ & & $\checkmark$ & & $\checkmark$ & $\checkmark$ \\
\hline
\end{tabular}

Table 1. Studied concerns in Pet Store

ing for interfaces names, clustering and unpluggability of the methods.

Further analysis of the application code, while keeping these indicators in mind, reveals that there are many classes/interfaces which implement/extend the Serializable interface. This is a common character of J2EE applications because of their distributed nature. From a logical point of view, this character does not really belong to the principal decomposition of the application [25] and therefore it could be treated as an aspect. An aspect capturing the serializable concern is the following:

declare parents : ProfileInfo implements Serializable; declare parents : HTMLAction extends Serializable; ...

where ProfileInfo is a class and HTMLAction is an interface. In Pet Store the purpose of using Serializable is simply to identify classes whose objects are serializable. This means the private writeobject and readObject methods are not customized and no field is marked as transient. Private methods cannot be introduced into target classes in AspectJ which means we would not be able to fully aspectize the serialization if Pet Store did customize the mentioned methods or used transient fields.

A total of 29 classes and 6 interfaces were declared to implement/extend the Serializable interface within this aspect. The explicit implements and extends declarations in the classes and interfaces were removed afterwards.

Further, EJB rules demand the extension of EJBLocalobject for the component interface and EJBLocalHome for the home interface. Pet Store has 13 component interfaces with a name ending in Local. There are also 13 home interfaces with a name ending in LocalHome. We have aspectized these two interface concerns as follows:

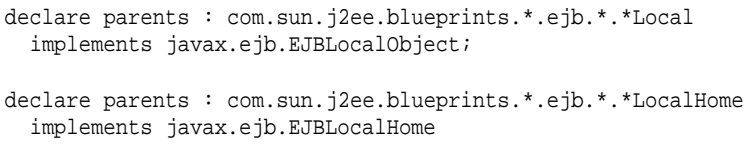

In this aspectized version, the two local EJB interfaces are modularized in a single aspect. The serialization concern is also handled in a single separate unit, resulting in an increased degree of modularization. This enables developers to get a better overall view of the classes implementing these interfaces.

\section{Discussion}

This section reflects on the results we obtained by studying the crosscutting nature of the top-down and bottom-up concerns. Table 1 shows an overview of the studied concerns in Pet Store. Each concern is examined against a set of properties:

Crosscutting Has the concern a crosscutting nature in the application? As it can be seen all the concerns are crosscutting in some way except for Security because of its welldesigned modular implementation.

Aspectizable From the crosscutting concerns, which ones are aspectizable? A concern is aspectizable when its crosscutting nature can be resolved by applying aspect-oriented refactoring. It is interesting to see that all the crosscutting concerns are aspectizable which shows that the aspectoriented paradigm is suited for tackling crosscutting problems.

Code Reduction Logic duplication is one of the manifestations of crosscutting concerns and aspect-oriented techniques are experts in minimizing that. Aspectizing the $E x$ ception Wrapping concern resulted in a $20 \%$ code reduction in the affected classes. Aspectizing the Precondition Checking concern allowed us to completely remove the check code from 9 classes, each class having one affected method. It is worth noting that only three of the aspectized concerns showed a reduction in code size. This implies that aspectoriented programming has a much broader purpose than only code reduction.

Obliviousness Obliviousness states that neither the existence nor the execution of the aspect code is apparent by examining the body of the base code. Obliviousness is desirable because it allows greater separation of concerns in the application development process $[7,6]$. Six of the aspectized concerns resulted in obliviousness. Persistence, however, shows only obliviousness but no solid evolvability improvement is evident in the Pet Store case. 
Reliability In certain cases, the use of an aspect makes it harder to make a particular kind of mistake. For example, the generic pointcut for the Exception Wrapping aspect ensures that new methods automatically have the naming exception wrapped into a ServiceLocator exception. For the same reason, faults are less likely for the Transaction and Precondition Checking concerns.

Modularity Aspectized modularity enables us to reduce tangling, multiple concerns intermixed, and scattering, spread of code for a concern, which in turn simplifies maintenance and evolution. Modularity obtained in four of the concerns in this case study has resulted in code that is more localized, less coupled, and has better cohesion. Transaction, for instance, was aspectized into a new module, enabling the removal of corresponding code from classes that were merely using Transaction, i.e., transaction management was not their primary function. Interface Migration is another example of achieving better modularity. The serialization concern was moved to an aspect, enabling us to remove the scattering code in all classes that need to be serializable. It has made the interface implementation transparent to the classes that were using it explicitly before the aspectization process. This allows us to add the serialization concern to new classes easily and also gives us a clear overview of all the classes using this particular concern, increasing design and code understandability.

Evolvability Software evolution is a process that either introduces new requirements into an existing system, or modifies the system if the requirements change or were not correctly implemented. We consider evolvability improvements by presenting possible change scenarios in the life-cycle of the application:

Transaction: Imagine we decide to use a different transaction management API. In the conventional implementation all classes using the transaction code had to be modified. In the new aspectized version, however, the change will take place in only one aspect. Further adding the functionality to a new class is a matter of expanding the pointcut in the transactional aspect.

Persistence: In case of the Persistence concern, it is questionable whether the Identifiable aspect (with the intention to achieve total obliviousness) provides us with any real evolvability benefits. This could imply that obliviousness alone is not enough to achieve better evolvability.

Exception Wrapping: Being able to modularize the Exception Wrapping concern allows us to have a uniform way of dealing with all the affected methods; i.e., there will be no inconsistencies in the type of wrapped exception thrown or the way exceptions are logged. The base code is separated from the aspect code which enables us to alter one independent of the other.

Precondition Checking: Precondition checking is one of those concerns with a volatile requirement that can easily change over time. If the requirement change states that, for instance, the check has to be extended on more parameters, it is now much easier to adapt the implementation.

ServiceLocator: A possible scenario is that we decide to refactor our ServiceLocator class not to be a Singleton anymore because Singleton makes it very hard to write unit tests, i.e, Singleton makes it very difficult to follow the testing independence rule. Because the application is now oblivious of the Singleton nature, this change will not affect the clients using the ServiceLocator class.

Interface Concern: We have a central module defining all the classes that implement a certain interface using aspects. We can also have a default implementation of the corresponding interface through aspects. Imagine we write a new class that needs to implement the interface. We then simply add the name of the new class to the list of the declared classes that implement the interface in the aspect. Interface Extraction has also given us the ability to make use of multiple inheritance if needed in the future.

Costs Utilizing aspect-oriented programming is not without costs. While aspects improve modularity, they can increase a system's complexity. For instance, the transaction concern is aspectized using an abstract aspect and a concrete aspect. Generally speaking, this is more complex to comprehend than the original simple implementation. Understandability is also at stake when aspects do not achieve full obliviousness. For example, a pointcut may rely on a particular naming pattern, which the developer, not aware of the aspect code, may break by applying a method renaming.

\section{Concluding Remarks}

Evaluation The key lessons learned from our experiments are the following. First, many concerns that are by nature crosscutting are well addressed by J2EE's container mechanism. Second, in those cases where the container mechanism is not sufficiently powerful or cannot be utilized, such as for the transaction mechanism adopted, an aspect-oriented implementation of these concerns does offer benefits. Third, crosscutting concerns in J2EE systems include generic ones as well, such as logging (not discussed), or precondition checking and interface extraction. Fourth, while adopting aspects in J2EE applications does have clear benefits, these are relatively small (as suggested by our example concerns and scenarios) and certainly not of an order of magnitude. Extrapolating some of the numbers we found, we suspect that there are approximately 25 reasonable aspect opportunities in Pet Store, on a total of 283 classes. We do expect, however, that in real life J2EE applications the need to circumvent J2EE's container mechanism will be much stronger (for example due to performance limitations), leading to significant benefits from the use of aspect-orientation in such cases. 
Contributions This paper has presented the results we have obtained by aspectizing a number of top-down and bottom-up crosscutting concerns in a J2EE case study. We have provided the full details of the resulting aspect-oriented code in order to outline a clear view of the advantages and disadvantages. The results show that even though proper use of design patterns and container-managed services helps encapsulation in many situations, we still find the repetition and concern-mixing phenomena even in well-designed J2EE systems. We have shown how aspect-oriented techniques provide a framework in which these crosscutting concerns can easily be modularized, allowing greater separation of concerns which in turn increases the evolvability of the system.

Future Work Our future work will focus on studying the crosscuttingness of concerns in a number of real world J2EE applications. We will try to apply a wider range of aspect mining techniques in order to identify aspect candidates. Our research will also examine the convenience of using other aspect-oriented tools (besides AspectJ) such as AspectWerkz ${ }^{4}$ in J2EE environments. We will also provide access to APetStore, an aspect-oriented refactoring of the Pet Store application, through our Web site, which will be further used to experiment with aspects.

\section{Acknowledgements}

We would like to thank Marius Marin and Magiel Bruntink for their reviews, advice and feedback. This paper received partial support from SenterNovem, project Single Page Computer Interaction (SPCI).

\section{References}

[1] D. Alur, J. Crupi, and D. Malks. Core J2EE Patterns. Sun Microsystems, Inc., USA, 2003.

[2] Jung Pil Choi. Aspect-Oriented Programming with Enterprise JavaBeans. In EDOC '00: Proceedings of the 4th International conference on Enterprise Distributed Object Computing, page 252. IEEE Computer Society, 2000.

[3] Tal Cohen and Joseph (Yossi) Gil. AspectJ2EE = AOP + J2EE: Towards an Aspect Based, Programmable and Extensible Middleware Framework. In ECOOP - Object-Oriented Programming, LNCS 3086, pages 219-243. Springer-Verlag, 2004 .

[4] A. van Deursen, M. Marin, and L. Moonen. Aspect mining and refactoring. In L. Thavildari and K. Kontogiannis, editors, Proceedings of the WCRE Workshop on REFactoring: Achievements, Challenges, Effects (REFACE03)., Waterloo, Canada, 2003. University of Waterloo.

[5] J. Fabry. Transaction management in EJBs: Better separation of concerns with AOP. In Y. Coady and D. Lorenz, editors, Proc. of the 3rd AOSD Workshop on Aspects, Components, and Patterns for Infrastructure Software (ACP4IS), Victoria, Canada, 2004. University of Victoria.

[6] Robert E. Filman. What Is Aspect-Oriented Programming, Revisited. Technical report, 2001.

[7] Robert E. Filman and Daniel P. Friedman. Aspect-Oriented Programming is Quantifi cation and Obliviousness. Technical report, 2000.

\footnotetext{
${ }^{4}$ http://aspectwerkz.codehaus.org/
}

[8] J. D. Gradecki and N. Lesiecki. Mastering AspectJ - Aspect Oriented Programmingin Java. Wiley Publishing, Inc., Indianapolis, Indiana, 2003.

[9] Minmin Han and Christine Hofmeister. Separation of Navigation Routing Code in J2EE Web Applications. In ICWE, pages 221-231, 2005.

[10] J. Hannemann and G. Kiczales. Design pattern implementation in Java and AspectJ. In Proceedings of the 17th Annual ACM conference on Object-Oriented Programming, Systems, Languages, and Applications (OOPSLA), pages 161173, Boston, MA, 2002. ACM Press.

[11] Mik Kersten and Gail C. Murphy. Atlas: a case study in building a web-based learning environment using aspect-oriented programming. In OOPSLA '99: Proceedings of the 14th ACM SIGPLAN conference on Object-oriented programming, systems, languages, and applications, pages 340-352. ACM Press, 1999.

[12] G. Kiczales, J. Lamping, A. Mendhekar, C. Maeda, C. Lopes, J-M. Loingtier, and J. Irwin. Aspect-oriented programming. In 11th Europeen Conf. Object-Oriented Programming, volume 1241 of LNCS, pages 220-242. Springer Verlag, 1997.

[13] H. Kim and S. Clarke. The relevance of AOP to an applications programmer in an EJB environment. In First International Conference on Aspect-Oriented Software Development (AOSD) Workshop on Aspects, Components, and Patterns for Infrastructure Software (ACP4IS), 2002.

[14] R. Laddad. Aspect-oriented refactoring. www.theserverside.com, December 2003 .

[15] R. Laddad. AspectJ in Action - Practical Aspect Oriented Programming. Manning Publications Co., Greenwich, CT, 2003.

[16] M. Lippert and C.V. Lopes. A study on exception detection and handling using aspect-oriented programming. In Proceedings of the 22nd International Conference on Software Engineering (ICSE), pages 418-427, Boston, MA, 2000. ACM Press.

[17] M. Marin, A. van Deursen, and L. Moonen. Identifying aspects using fan-in analysis. In Proceedings of the 11th Working Conference on Reverse Engineering (WCRE2004)., pages 132-141. IEEE Computer Society, 2004.

[18] T. Murali, R. Pawlak, and H. Younessi. Applying aspect orientation to J2EE business tier patterns. In Y. Coady and D. Lorenz, editors, Proc. of the 3rd AOSD Workshop on Aspects, Components, and Patterns for Infrastructure Software (ACP4IS), Victoria, Canada, 2004. University of Victoria.

[19] Odysseas Papapetrou and George A. Papadopoulos. Aspect oriented programming for a component-based real life application: a case study. In SAC '04: Proceedings of the 2004 ACM Symposium on Applied Computing, pages 1554 1558. ACM Press, 2004.

[20] Awais Rashid and Ruzanna Chitchyan. Persistence as an aspect. In AOSD '03. Proceedings of the 2nd international conference on Aspect-oriented software development, pages 120-129. ACM Press, 2003.

[21] Bill Shannon, Mark Hapner, Vlada Matena, James Davidson, James Davidson, and Larry Cable, editors. Java 2 Platform, Enterprise Edition: Platform and Component Specifi cations. Pearson Education, 2000.

[22] Inderjeet Singh, Beth Stearns, and Mark Johnson. Designing Enterprise Applications with the J2EE Platform, Second Edition. Java BluePrints Guidelines. java.sun.com/blueprints/guidelines.

[23] P. Slowikowski and K. Zielinski. Comparison study of aspect-oriented and container managed security. In AAOS2003: Analysis of Aspect Oriented Software. Workshop held in conjunction with ECOOP, 2003.

[24] Sergio Soares, Eduardo Laureano, and Paulo Borba. Implementing distribution and persistence aspects with AspectJ. In OOPSLA '02: Proceedings of the 17th ACM SIGPLAN conference on Object-oriented programming, systems, languages, and applications, pages 174-190. ACM Press, 2002.

[25] Paolo Tonella and Mariano Ceccato. Migrating interface implementation to aspects. In Proceedings of the 20th IEEE International Conference on Software Maintenance (ICSM'04), pages 220-229. IEEE Computer Society, 2004.

[26] C. Zhang and H-A. Jacobsen. Quantifying aspects in middleware platforms. In Proc. 2nd Int. Conf. on Aspect-Oriented Software Development (AOSD-2003), pages 130-139. ACM Press, March 2003. 